\title{
Cholinergic neurons mediate CaMKII-dependent enhancement of courtship suppression
}

\author{
Jennifer E. Mehren ${ }^{1}$ and Leslie C. Griffith ${ }^{2}$ \\ Brandeis University, Department of Biology and Volen Center for Complex Systems, Waltham, Massachusetts 02454-9110, USA
}

\begin{abstract}
In Drosophila, calcium/calmodulin-dependent protein kinase II (CaMKII) activity is crucial in associative courtship conditioning for both memory formation and suppression of courtship during training with a mated female. We have previously shown that increasing levels of constitutively active CaMKII, but not calcium-dependent CaMKII, in a subset of neurons can decrease the initial level of courtship and enhance the rate of suppression of courtship in response to a mated female. In this study, we demonstrate that a subpopulation of noncholinergic, nondopaminergic, non-GABAergic neurons can cause CaMKII-dependent reductions in initial courtship, but only cholinergic neurons enhance training-dependent suppression. These data suggest that processing of pheromonal signals in two subpopulations of neurons, likely antennal lobe projection neurons, is critical for behavioral plasticity.
\end{abstract}

Modification of behavior on short time scales requires fast, likely cell signaling, changes in neuronal circuits. One molecule that has been shown in many systems to be responsible for both short-term and long-term changes in neuronal activity is calcium/calmodulin-dependent protein kinase II (CaMKII). This kinase can act a molecular switch, becoming calcium independent after autophosphorylation at T287. Production of constitutively active kinase is believed to be a first step toward establishing short- and long-term changes in neuronal properties that underlie learning (for review, see Lisman et al. 2002).

In Drosophila courtship conditioning, CaMKII is important for behavioral changes that occur during the training period and for formation of associative memory (Griffith et al. 1993; Joiner and Griffith 1997, 1999). In this behavioral paradigm, a male is exposed to a previously mated female for $1 \mathrm{~h}$, and the effects of training are assessed by measuring courtship of a subsequently presented virgin female. Naive males exposed to virgin females sense female-specific stimulatory pheromones and rapidly initiate courtship, usually copulating within $15 \mathrm{~min}$. In comparison, trained males show reduced courtship of virgins, and this is believed to be the result of association of stimulatory pheromones with an aversive substance given off by mated females (Tompkins et al. 1983). Memory formation can be blocked by inhibition of CaMKII in mushroom bodies, central complex, and parts of the lateral protocerebrum (Joiner and Griffith 1999). The behavior of males during training is also plastic; they initiate vigorous courtship, but over the course of training they decrease their intensity of courtship and rarely copulate. The suppression of courtship during training is driven by a neuronal circuit distinct from that mediating associative memory formation, involving neurons in the antennal lobes and lateral protocerebrum (Joiner and Griffith 1999).

The requirement for CaMKII activity was determined using spatially restricted expression of a peptide, which inhibits both the calcium-dependent and the calcium-independent (autophosphorylated at T287) forms of the kinase. The role of these two forms of CaMKII was dissected by spatially and temporally regu-

1Present address: Laboratory of Neurogenetics and Behavior, The Rockefeller University, New York, New York 10021-6399, USA. ${ }^{2}$ Corresponding author.

E-mail griffith@brandeis.edu; fax (781) 736-3107.

Article published online before print. Article and publication date are at http:// www.learnmem.org/cgi/doi/10.1101/lm.317806. lated overexpression of T287A and T287D CaMKII point mutants (Mehren and Griffith 2004). Expression of the constitutively active T287D CaMKII enhanced the suppression of courtship during training but had no effect on memory formation. Addition of T287A CaMKII, which is incapable of becoming calcium independent, had no behavioral effects. The effect of T287D CaMKII on courtship suppression was only seen in animals that expressed T287D in subsets of the adult brain defined by the $30 Y$ GAL4 pattern, which includes the mushroom bodies, lateral protocerebrum, antennal lobes, subesophageal ganglion, and optic lobes. These results suggested that the decision to court was not sensitive to the total amount of calcium-stimulable kinase but rather to some threshold level of constitutively active CaMKII.

In this study, we demonstrate that both initial and trainingdependent changes in courtship respond to the level of calciumindependent CaMKII in a dose-dependent manner. We further show that initial courtship levels can be modulated by noncholinergic neurons, whereas the effects of T287D on trainingdependent changes in courtship are mediated by cholinergic antennal lobe neurons.

\section{Results and Discussion}

To determine whether courtship suppression during training was sensitive to the absolute level of constitutively active CaMKII, we expressed T287D CaMKII under control of a tripartite GAL4/UAS/ tet-off driver system (Mehren and Griffith 2004). This system allows for both spatial and temporal control of the level of transgene expression. The tetracycline transactivator (UAS-tTA) is driven by GAL4 (a yeast transcription factor that binds to the UAS element and drives transcription of UAS-linked genes) in a spatially restricted pattern. The spatial restriction of GAL4 is achieved by expressing it under control of particular defined or endogenous promoter sequences. The effector transgene (tetOT287D) in this scheme is expressed in the GAL4 pattern, but its levels can be controlled by feeding the animal tetracycline, which inhibits transcription by tTA. This system allows complete suppression of toxic transgenes during development by growing larvae on $10 \mu \mathrm{g} / \mathrm{mL}$ tetracycline in the food agar. In adults, complete suppression can be achieved by feeding $100 \mu \mathrm{g} / \mathrm{mL}$ tetracycline in sucrose. Removal or reduction of the drug leads to induction of the transgene.

Previous work had shown that expressing T287D with tetracycline-controlled 30Y-GAL4 and allowing maximal adult in- 
duction by placing newly eclosed males on food containing no tetracycline was able to cause both a lowered level of initial courtship and an enhancement of trainer-dependent suppression (Mehren and Griffith 2004). If the mechanism of calciumindependent CaMKII's actions was dependent on the level of calcium-independent CaMKII, we would expect that manipulating the amount of induction by placing newly eclosed males on a range of drug concentrations from 100 to $0 \mu \mathrm{g} / \mathrm{mL}$ should produce a dose-dependent range of levels of courtship suppression. Figure 1 shows that after rearing on a maximally suppressing dose of tetracycline, suppression scales with the adult level of tetracycline. Initial courtship (Fig. 1A) is decreased with increasing expression of T287D and the animals fall into two groups that are statistically different: Untreated wild-type males, fully suppressed $(100 \mu \mathrm{g} / \mathrm{mL}$ tetracycline) T287D males, and T287D males maintained on $10 \mu \mathrm{g} / \mathrm{mL}$ tetracycline all show high initial courtship, whereas animals on 1 or $0 \mu \mathrm{g} / \mathrm{mL}$ tetracycline have a similar, low level of courtship. This suggests that there is a bimodal relationship between the level of constitutive CaMKII and initiation of courtship.

The drop in courtship caused by continued exposure to the mated female is also sensitive to the level of CaMKII. Males with

A
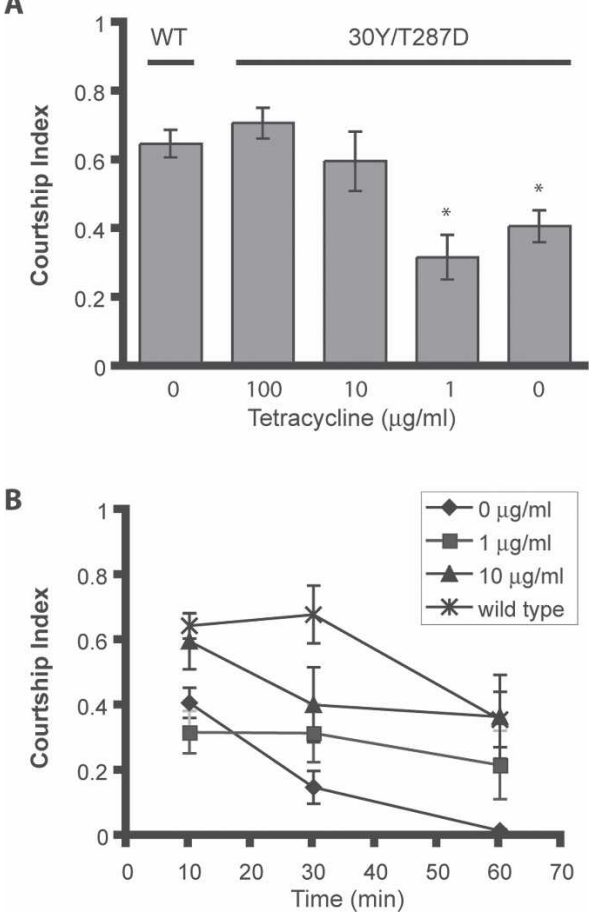

Figure 1. Initial courtship levels are dependent on the amount of T287D CaMKII expressed. tetO-T287D ${ }^{7.1} /+; 30 Y-G A L 4 / U A S-t T A^{221}$ flies were grown on a maximum dose of tetracycline to suppress T287D CaMKII expression completely during development, then moved upon eclosion to different doses of tetracycline to get a range of levels of T287D CaMKII expression. As a control, wild-type flies were treated the same as larvae, and transferred to $0 \mu \mathrm{g} / \mathrm{mL}$ tetracycline as adults. $(A)$ Dose dependence of initial courtship for T287D CaMKII flies. Flies expressing 30Y-driven T287D CaMKII at low levels (fed higher concentrations of tetracycline as adults, 10 or $100 \mu \mathrm{g} / \mathrm{mL}$ ) show levels of initial courtship similar to wild type, whereas flies expressing higher levels of T287D CaMKII as adults (fed 0 or $1 \mu \mathrm{g} / \mathrm{mL}$ tetracycline as adults) show levels of initial courtship significantly lower than wild type (ANOVA $F_{[4,78]}=9.21$, $P<0.0001$, with post hoc comparisons for $30 Y / T 287 D$ fed 0 or $1 \mu \mathrm{g} / \mathrm{mL}$ tetracycline compared to wild type significantly different, $\alpha=0.013)$. (B) Dose dependence of the time course of courtship suppression for T287D CaMKII flies. $n=10-24$. additional T287D CaMKII begin to suppress courtship immediately without the characteristic lag seen in wild-type animals (Fig. 1B) (Mehren and Griffith 2004). But in contrast to the effects of T287D dosage on initial courtship, the effects on the trainerspecific component are more graded over the range of doses administered. Animals expressing the maximum level of T287D (fed $0 \mu \mathrm{g} / \mathrm{mL}$ tetracycline as adults) suppress courtship toward the mated female $\left(\mathrm{CI}_{\mathrm{f}} / \mathrm{CI}_{\mathrm{in}}=0.04 \pm 0.04\right.$, data presented as mean \pm SEM) 12 -fold better than animals fed $10 \mu \mathrm{g} / \mathrm{mL}$ tetracycline $\left(\mathrm{CI}_{\mathrm{f}} / \mathrm{CI}_{\text {in }}=0.47 \pm 0.14\right)$, and 18 -fold better than fully suppressed animals (fed $100 \mu \mathrm{g} / \mathrm{mL}$ tetracycline $\left[\mathrm{CI}_{\mathrm{f}} /\right.$ $\left.\mathrm{CI}_{\mathrm{in}}=0.70 \pm 0.09\right]$ ). The difference in the effects of particular doses of T287D on initial and trainer-dependent suppression suggests that these effects may be mediated by distinct sets of cells that innervate the antennal lobe.

To refine the mapping of these two types of courtship suppression, we utilized GAL4 lines that are restricted to neurons expressing particular neurotransmitters. The antennal lobe is known to contain synapses made by cholinergic olfactory receptor neurons (Barber et al. 1989), cholinergic projection neurons (Yasuyama et al. 2003), GABAergic interneurons (Ng et al. 2002; Wilson and Laurent 2005) and perhaps other neurons that release modulatory peptides or amines (Python and Stocker 2002). By restricting T287D expression to adult neurons of particular neurochemical classes, we can subdivide the antennal lobe circuit. Figure $2 \mathrm{~A}$ shows the expression patterns of Cha-GAL4 (restricted to cholinergic neurons), TH-GAL4 (restricted to dopaminergic neurons), GAD-GAL4 (restricted to GABAergic neurons) as assessed by mCD8-GFP expression. All of these neurochemical GAL4s show antennal lobe expression.

We can also neurochemically subdivide the 30Y-GAL4 circuitry using GAL80 (Lee and Luo 1999). GAL80 is a yeast protein that antagonizes GAL4 action. Expression of GAL80 under control of the Cha promoter will block GAL4 action exclusively in the cholinergic subpopulation of a particular GAL4 pattern (Kitamoto 2002). Figure $2 \mathrm{~B}$ shows the effect of adding Cha-GAL80 to $30 Y-G A L 4$. All antennal lobe expression of UAS-mCD8-GFP is lost, but substantial expression remains in the mushroom body, pars intercerebralis, and extrinsic mushroom body neurons, indicating that these neurons are not cholinergic.

To assess the contribution of these neurons to the T287Ddependent decrease in initial courtship, we examined the ratio of courtship during the first $10 \mathrm{~min}$ of exposure to a mated female $\left(\mathrm{CI}_{\mathrm{in}}\right)$ of males with adult expression of constitutively active CaMKII in neurochemical subsets of cells to the mean of their GAL4 driver control (Fig. 3A). $\mathrm{CI}_{\text {in }} \mathrm{s}$ for effector transgene controls (tetO-T287D/+; UAS-tTA/+, $0.51 \pm 0.05$; UAS-tTA/+; ChaGAL80/+, $0.68 \pm 0.05)$ did not differ significantly from those of wild type $(0.60 \pm 0.05)$ (data presented as mean \pm SEM and analyzed by ANOVA $\left.F_{[2,64]}=2.78, P=0.07\right)$. A ratio of less than one indicates a T287D-specific decrease in initial courtship and controls for nonspecific effects of individual GAL4 drivers. Consistent with our observations in Figure 1A, initial levels of courtship in animals expressing T287D under control of 30Y-GAL4 are significantly lower than the GAL4 control. Interestingly, TH-GAL4, Cha-GAL4, and GAD-GAL4 drivers cannot cause a T287D-specific decrease in initial courtship. In agreement with this, removal of the cholinergic component of the $30 Y$ pattern by coexpression of Cha-GAL80 does not alter the effects of T287D, indicating that the cells marked by $30 Y$ that are controlling initial courtship are noncholinergic.

Training-dependent courtship suppression can also be dissected neurochemically by looking at the training index $\left(\mathrm{CI}_{\mathrm{f}} /\right.$ $\mathrm{CI}_{\mathrm{in}}$ ) normalized to the training index of the GAL4 control. Again, a ratio of less than one indicates a T287D-specific effect on trainer-dependent suppression. Animals expressing T287D under 

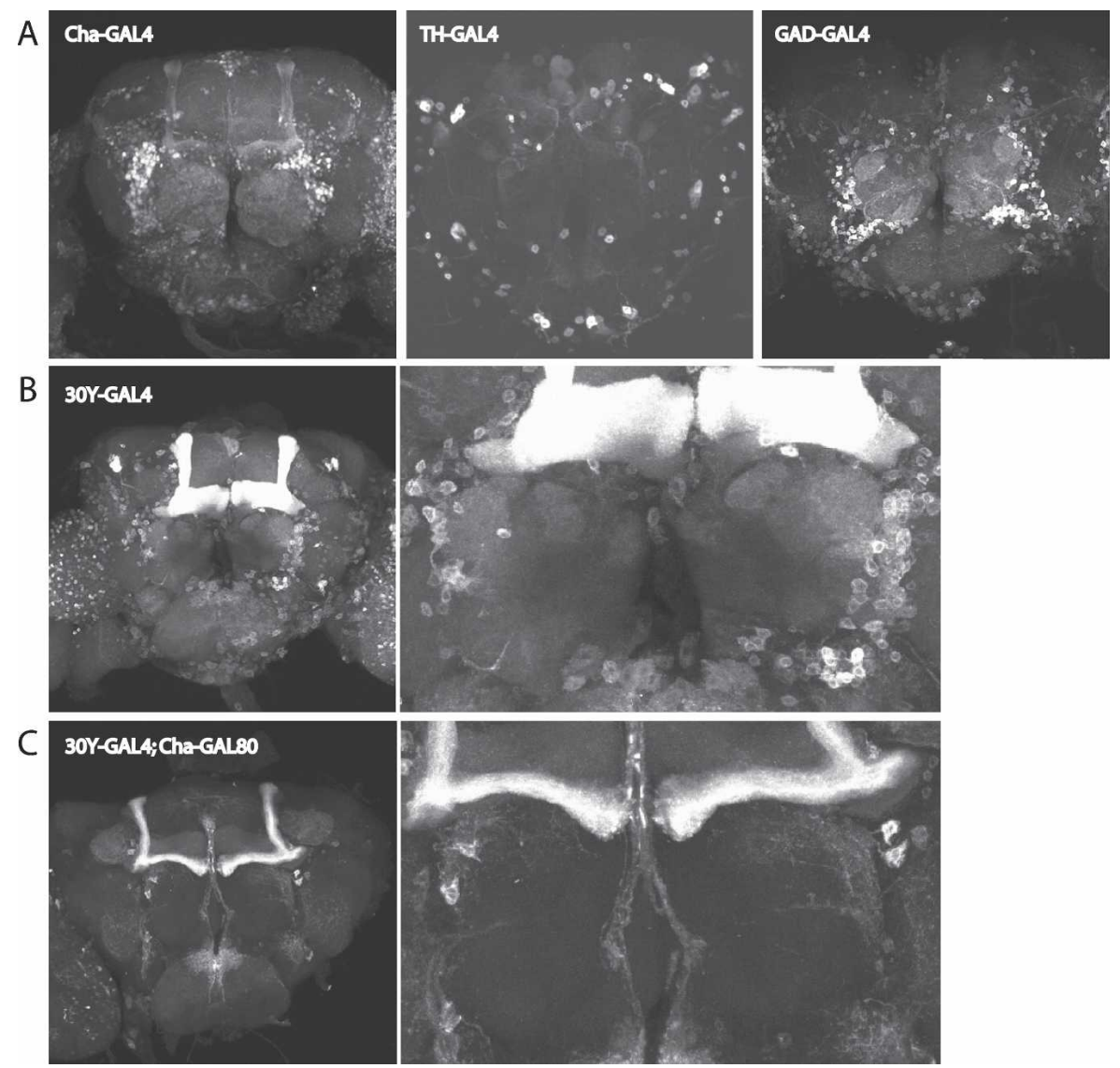

Figure 2. Expression patterns of neurochemical GAL4 drivers. Expression of UAS-mCD8-GFP was visualized in whole-mount adult brains. Fluorescent images were collected as z-stacks on a Leica TCS SP2 confocal scanning microscope. (A) Expression of UAS-mCD8-GFP under control of Cha-GAL4 (left), TH-GAL4 (middle), and GAD-GAL4 (right). (B, C) Adding Cha-GAL80 to the 30Y-GAL4 pattern reduces the number of neurons labeled in the adult brain. UAS-mCD8GFP was driven by $30 Y-G A L 4(B)$ or by $30 Y$-GAL4 with Cha-GAL80 present (C). For B and C, whole-brain confocal stacks are shown on the left; enlarged images of the antennal lobes are shown on the right.

control of 30Y-GAL4 and Cha-GAL4 show an enhanced suppression (Fig. 3B). TH-GAL4, GAD-GAL4, and 30Y-GAL4/Cha-GAL80 do not enhance training when driving T287D. Effector transgene controls (tetO-T287D/+; UAS-tTA/+, $0.76 \pm 0.17$; UAS-tTA/+; Cha-GAL80/+, $0.73 \pm 0.11$ ) did not differ significantly from wild type $(0.55 \pm 0.12)$ (data presented as mean \pm SEM and analyzed by ANOVA $F_{[2,64]}=0.95, P=0.39$ ). These data suggest that the changes in trainer-specific suppression of courtship caused by T287D are mediated by its expression in cholinergic neurons.

Our data suggest that the ability of constitutively active CaMKII to promote suppression of courtship derives from its actions in two separable populations of cells, both likely innervating the antennal lobes. Initial courtship levels are modulated by CaMKII activity in a population of noncholinergic neurons. These neurons are very sensitive to the levels of calciumindependent CaMKII.

Plasticity of courtship during exposure to a mated female is responsive to CaMKII levels in a different set of cells. Expression of T287D in cholinergic inputs into the antennal lobes driven by either $30 Y-G A L 4$ or Cha-GAL4 is capable of accelerating the trainer-dependent suppression that occurs in response to pheromonal signals that mated females emit. These cells are sensitive to graded increases in constitutive CaMKII activity. Both olfactory sensory neurons and projection neurons are cholinergic and innervate the antennal glomeruli. Since T287D expression in primary sensory neurons does not produce an enhancement of training (Mehren and Griffith 2004), the most likely antennal cholinergic neurons to be responsible for this plasticity effect are the projection neurons. We have also previously shown that $30 Y-G A L 4$ does not express in antennae (Mehren and Griffith 2004) where primary olfactory neurons reside, supporting the idea that the CaMKIIsensitive cholinergic neurons of the $30 Y$ GAL4 pattern are projection neurons. This makes it likely that projection neurons are active participants in CaMKIIdependent short-term plasticity of courtship behavior.

\section{Materials and Methods}

\section{Fly lines}

Fly stocks and crosses were maintained on cornmeal, yeast, dextrose, and agar medium and kept at $25^{\circ} \mathrm{C}$ in a 12 -h light/12-h dark cycle. Tetracycline feeding was performed by growing larvae on medium containing tetracycline at 10 $\mu \mathrm{g} / \mathrm{mL}$ food or by feeding tetracycline to adults at a concentration of $0,1,10$, or $100 \mu \mathrm{g} / \mathrm{mL}$ in a $4 \%$ sucrose solution, as described in Mehren and Griffith (2004). Canton S flies were used as wild type, and all transgenic lines were in a Canton $\mathrm{S}$, white background. UAS-tTA was a gift from Bruno Bello (University of Basel, Basel, Switzerland). The tetO-CaMKIIT287D line was described in Mehren and Griffith (2004). The lines 30Y-GAL4 (Yang et al. 1995), Cha-GAL4 (Salvaterra and Kitamoto 2001), Cha-GAL80 (Kitamoto 2002) and TH4B-GAL4 (FriggiGrelin et al. 2003) have been previously described. GAD2B-GAL4 was a generous gift of Toshihiro Kitamoto (University of Iowa, Iowa City, IA).

\section{Immunohistochemistry}

Brains were dissected in PBS from 5- or 6-d-old adult flies, fixed in $4 \%$ paraformaldehyde for $20-30 \mathrm{~min}$, washed in PBS, and mounted in glycerol and Vectashield (Vector Laboratories Inc.). Images were acquired using the $40 \times$ objective on a Leica TCS SP2 confocal scanning microscope.

\section{Behavior}

All behavioral observations were performed at $25^{\circ} \mathrm{C}$ and $70 \%$ relative humidity in a Harris Environmental Room, in dim red light, as described in Mehren and Griffith (2004). Courtship conditioning was carried out by placing individual males with fertilized females (mated the day before) in Plexiglas mating chambers ( $8 \mathrm{~mm}$ diameter, $3 \mathrm{~mm}$ high) for $1 \mathrm{~h}$. Courtship indices (CIs) were measured for 3 time intervals during training $\left(0-10 \mathrm{~min}\left[\mathrm{CI}_{\mathrm{in}}\right]\right.$, 20-30 min, and 50-60 min $\left.\left[\mathrm{CI}_{\mathrm{f}}\right]\right)$. A training index was taken as the fraction of the $\mathrm{CI}_{\mathrm{f}}$ over the $\mathrm{CI}_{\mathrm{in}}$. Flies who had $\mathrm{CI}_{\mathrm{in}} \mathrm{s}<0.1$ were considered "nonbehaving" and therefore were excluded from analysis. Each CI was subjected to arcsine or arcsine square root transformation to approximate normal distributions. Transformed CIs or training indices were subjected to a one-way ANOVA with genotype as the main effect, using JMP (version 5.0.1.2; SAS Institute), and planned comparisons of means were performed with Student's $t$-test (adjusted for experiment-wise error).

\section{Learning \& Memory


A

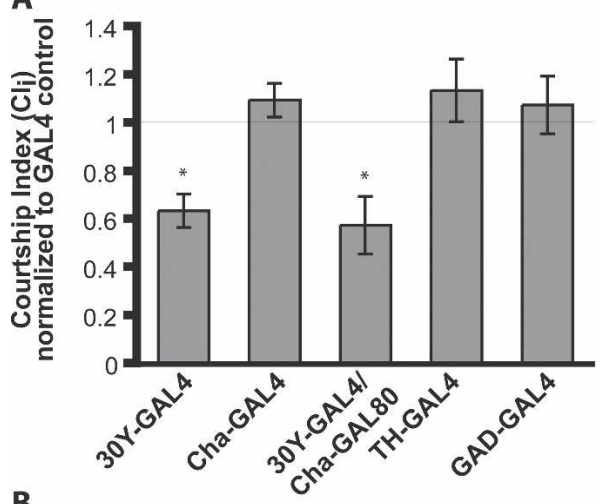

B

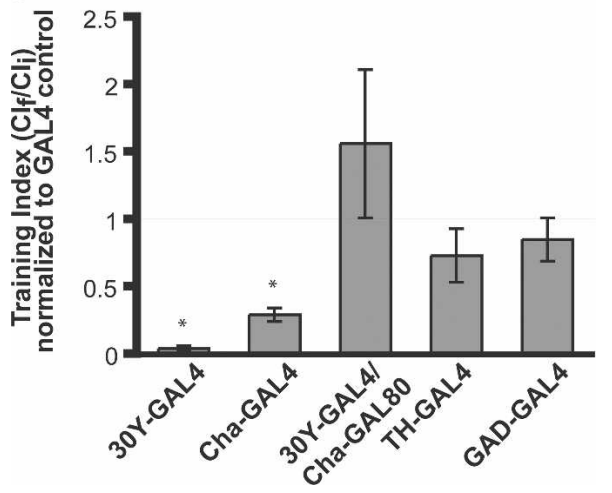

Figure 3. Neurochemical restriction separates initial and trainerdependent effects of T287D CaMKII. In all experiments T287D CaMKII expression was limited to adult neurons by use of the GAL4/UAS/tetoff system with $10 \mu \mathrm{g} / \mathrm{mL}$ tetracycline during development and no tetracycline feeding in the adult stage. $(A)$ Initial courtship levels are modulated by expression of T287D CaMKII in noncholinergic cells. $\mathrm{Cl}_{\text {in }}$ data for T287D CaMKII-expressing males was normalized to the mean of the $\mathrm{Cl}_{\text {in }}$ for the appropriate GAL4 line. A normalized ratio of $<1.0$ means that T287D CaMKII reduced initial courtship. Flies expressing $30 Y$ - or 30Y/Cha-GAL80-driven T287D CaMKII show significantly less initial courtship of mated females than do GAL4 controls (ANOVA $F_{[8,147]}=3.37, P=0.0014$, with post hoc comparisons significantly different, $\alpha=0.01$ ). (B) Cholinergic cells mediate T287D-dependent enhancement of courtship training. Training index $\left(\mathrm{Cl}_{\mathrm{f}} / \mathrm{Cl}_{\text {in }}\right)$ data for T287D CaMKII-expressing males was normalized to the mean of the training index for the appropriate GAL4 line. A normalized ratio of $<1.0$ means that T287D CaMKII enhanced training-dependent courtship suppression. When 30Y-GAL/4 is used to drive T287D CaMKII expression in adults only, training performance is enhanced, however when ChaGAL80 is used to inhibit expression of T287D in cholinergic cells of the $30 Y-G A L 4$ pattern, training enhancement is rescued. When T287D is expressed solely in cholinergic cells using Cha-GAL4, training enhancement is similar to enhancement with $30 \mathrm{Y}$-driven T287D. CaMKII expression in dopaminergic or GABAergic cells in the adult brain does not significantly affect courtship training with a mated female. ANOVA $F_{[8,146]}=5.03$, $P<0.0001$, with post hoc comparisons between 30Y-GAL4/T287D or Cha-GAL4/T287D, and GAL4 controls significantly different, $\alpha=0.01$. $n=9-22$.

\section{Acknowledgments}

This work was supported by NIH grants P01 NS44232 to L.C.G. and F32 NS43024 to J.E.M. We thank Mark Mattaliano for images of TH-GAL4 and Ed Dougherty for general help with imaging. The Brandeis Confocal Facility is supported by NIH grants P30 NS045713 and S10 RR16780. We thank Toshihiro Kitamoto for generously sharing unpublished fly lines with us.

\section{References}

Barber, R.P., Sugihara, H., Lee, M., Vaughn, J.E., and Salvaterra, P.M. 1989. Localization of Drosophila neurons that contain choline acetyltransferase messenger RNA: An in situ hybridization study. $J$. Comp. Neurol. 280: 533-543.

Friggi-Grelin, F., Coulom, H., Meller, M., Gomez, D., Hirsh, J., and Birman, S. 2003. Targeted gene expression in Drosophila dopaminergic cells using regulatory sequences from tyrosine hydroxylase. I. Neurobiol. 54: 618-627.

Griffith, L.C., Verselis, L.M., Aitken, K.M., Kyriacou, C.P., and Greenspan, R.J. 1993. Inhibition of calcium/calmodulin-dependent protein kinase in Drosophila disrupts behavioral plasticity. Neuron 10: 501-509.

Joiner, M.A. and Griffith, L.C. 1997. CaM kinase II and visual input modulate memory formation in the neuronal circuit controlling courtship conditioning. J. Neurosci. 17: 9384-9391.

Joiner, M.A. and Griffith, L.C. 1999. Mapping of the anatomical circuit of CaM kinase-dependent courtship conditioning in Drosophila. Learn. Mem. 6: 177-192.

Kitamoto, T. 2002. Conditional disruption of synaptic transmission induces male-male courtship behavior in Drosophila. Proc. Natl. Acad. Sci. 99: 13232-13237.

Lee, T. and Luo, L. 1999. Mosaic analysis with a repressible cell marker for studies of gene function in neuronal morphogenesis. Neuron 22: $451-461$.

Lisman, J., Schulman, H., and Cline, H. 2002. The molecular basis of CaMKII function in synaptic and behavioural memory. Nat. Rev. Neurosci. 3: 175-190.

Mehren, J.E. and Griffith, L.C. 2004. Calcium-independent calcium/calmodulin-dependent protein kinase II in the adult Drosophila CNS enhances the training of pheromonal cues. J. Neurosci. 24: 10584-10593.

Ng, M., Roorda, R.D., Lima, S.Q., Zemelman, B.V., Morcillo, P., and Miesenbock, G. 2002. Transmission of olfactory information between three populations of neurons in the antennal lobe of the fly. Neuron 36: 463-474.

Python, F. and Stocker, R.F. 2002. Immunoreactivity against choline acetyltransferase, $\gamma$-aminobutyric acid, histamine, octopamine, and serotonin in the larval chemosensory system of Drosophila melanogaster. J. Comp. Neurol. 453: 157-167.

Salvaterra, P.M. and Kitamoto, T. 2001. Drosophila cholinergic neurons and processes visualized with Gal4/UAS-GFP. Brain Res. Gene Expr. Patterns 1: 73-82.

Tompkins, L., Siegel, R.W., Gailey, D.A., and Hall, J.C. 1983. Conditioned courtship in Drosophila and its mediation by association of chemical cues. Behav. Genet. 13: 565-578.

Wilson, R.I. and Laurent, G. 2005. Role of GABAergic inhibition in shaping odor-evoked spatiotemporal patterns in the Drosophila antennal lobe. J. Neurosci. 25: 9069-9079.

Yang, M.Y., Armstrong, J.D., Vilinsky, I., Strausfeld, N.J., and Kaiser, K. 1995. Subdivision of the Drosophila mushroom bodies by enhancer-trap expression patterns. Neuron 15: 45-54.

Yasuyama, K., Meinertzhagen, I.A., and Schurmann, F.W. 2003. Synaptic connections of cholinergic antennal lobe relay neurons innervating the lateral horn neuropile in the brain of Drosophila melanogaster. J. Comp. Neurol. 466: 299-315.

Received May 11, 2006; accepted in revised form July 18, 2006. 


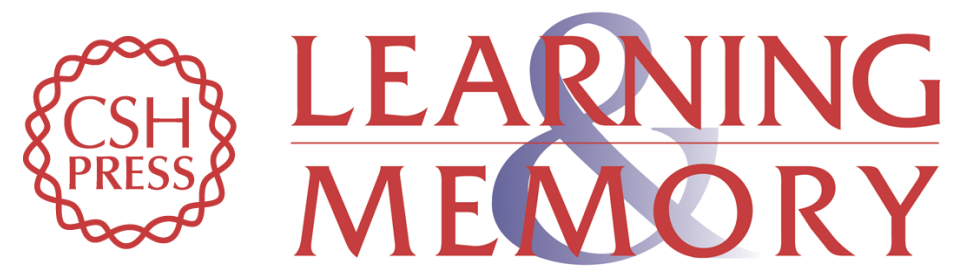

\section{Cholinergic neurons mediate CaMKII-dependent enhancement of courtship suppression}

Jennifer E. Mehren and Leslie C. Griffith

Learn. Mem. 2006, 13: originally published online November 13, 2006

Access the most recent version at doi:10.1101/lm.317806

References This article cites 16 articles, 5 of which can be accessed free at:

http://learnmem.cshlp.org/content/13/6/686.full.html\#ref-list-1

License

Email Alerting Receive free email alerts when new articles cite this article - sign up in the box at the Service top right corner of the article or click here. 